\title{
Reading Images and Seeing Words. Edited by Alan English and Rosalind Silvester
}

\section{Luigi Luison}

\section{Q OpenEdition}

1 Journals

\section{Edizione digitale}

URL: http://journals.openedition.org/studifrancesi/34042

DOI: $10.4000 /$ studifrancesi.34042

ISSN: 2421-5856

\section{Editore}

Rosenberg \& Sellier

\section{Edizione cartacea}

Data di pubblicazione: 1 décembre 2005

Paginazione: 703-704

ISSN: 0039-2944

\section{Notizia bibliografica digitale}

Luigi Luison, «Reading Images and Seeing Words. Edited by Alan English and Rosalind Silvester», Studi Francesi [Online], 147 (XLX | III) | 2005, online dal 30 novembre 2015, consultato il 19 avril 2021. URL: http://journals.openedition.org/studifrancesi/34042; DOI: https://doi.org/10.4000/studifrancesi. 34042

Questo documento è stato generato automaticamente il 19 avril 2021.

\section{(c)}

Studi Francesi è distribuita con Licenza Creative Commons Attribuzione - Non commerciale - Non opere derivate 4.0 Internazionale. 


\title{
Reading Images and Seeing Words. Edited by Alan English and Rosalind Silvester
}

\author{
Luigi Luison
}

\section{NOTIZIA}

AA.VV., Reading Images and Seeing Words. Edited by ALAN ENGLISH and ROSALIND SILVESTER, Amsterdam, Editions Rodopi, 2004, pp. 188.

1 La doppia natura insita nel titolo di questa raccolta si propone di far convergere tra loro le duplici strategie coinvolte nella produzione del significato: forme narrative che sono a priori non-narrative e la possibilità di forme visive che sono all'origine nonvisive. Se da una parte vi è l'evidente divergenza tra immagine e parola, dall'altra c'è un'evidente convergenza ogni qualvolta la forma illustrata può essere vista comportarsi come forma verbale, dove le parole sono letteralmente un tutt'uno con l'immagine.

2 Ogni saggio della prima parte di questo volume, intitolato Reading Images, analizza in qualche modo queste due tendenze, chiedendosi se l'immagine sia relativizzata dalla parola o viceversa. Nei vari lavori di questa sezione l'immagine precede il testo e qualche volta riproduce anche il suo carattere linguistico, lineare e articolato.

3 È noto come all'inizio del ventesimo secolo sia stato riconosciuto che la parola crea e costituisce la propria plasticità o realtà e che questo processo trova il suo parallelo nelle moderne tecniche artistiche che hanno a che fare con l'immagine fissa (pittura, disegno, etc.) o con l'immagine animata (film, video, etc.). In questo contesto si inserisce il saggio di Pina PARISH (Henri Michaux: Destruction of the Book Form and Creation of the Book-Object, pp. 19-36) sulle opere di Henri Michaux. L'A. parte dall'uso che Michaux fa dello spazio bianco, ritenendo che esso punteggi lo scritto in modo ritmico, separando sequenze di figure diverse. L'A. analizza sia i tratti neri, che alludono a 
qualcosa di indefinito, ad un codice semantico che non è stato definito e che non può, perciò, essere convenzionale, sia l'uso raro dell'inchiostro rosso, il cui significato simbolico allude al colore della morte e della violenza. Il ricorso alle immagini da parte di Michaux, suggerisce un'alternativa in tono con la condizione moderna: un libro che ci porta a guardare come possiamo leggere e come possiamo esprimere esperienze personali all'interno di una struttura universale.

4 Il Cubismo è la direzione verso cui si orienta il saggio di Nicola CREIGHTON (The Revolution of the Word follows the Revolution of the Eye: Carl Einstein and Cubism in Image and Text, pp. 37-56). Partendo dall'idea che l'osservatore rimane statico e neutrale, l'A. si sofferma, invece, sul processo dinamico e creativo del "vedere". In particolare vengono enunciate le convinzioni del critico d'arte Einstein in relazione alla cosiddetta quarta dimensione.

5 I saggi di Maria WALSH (Narrative Duration: Tacita Dean's “ Disappearance at Sea”, pp. 57-69) e Guillaume thouroude (Une ligne: Proust...Beckett...Deleuze. L'image épuisante, pp.71-81) individuano in particolare, un filo rosso che unisce la metafora sperimentale di Proust al rapporto con l'immagine secondo Beckett e Deleuze.

6 Sul rapporto parola/immagine, Massimo LEONE (Words, Images and Knots, pp. 83-106) sostiene che le rappresentazioni visive e verbali possono essere entrambe studiate come testi in cui le immagini possono essere considerate come formate da "linee nodi", a differenza del testo scritto che, etimologicamente parlando, può essere considerato semplicemente un "gruppo di nodi". L'A. conferma l'idea che l'immagine assembla e coordina diverse categorie di segno.

7 Nella seconda parte del volume, intitolata Seeing Words, Rosalind SILVESTER (Seeing is Perceiving: Sartre's Language of Phenomenology, pp. 109-122) spiega che il modo in cui Sartre utilizza il linguaggio per descrivere la percezione fenomenologica ha alcuni elementi in comune e altri in opposizione con la fenomenologia di Husserl. Così come Sartre separa i regni dell'immaginazione e della percezione, anche l'uomo si muove necessariamente nell'immaginario lasciando dietro di sè l'atto di pura percezione.

In relazione al tema centrale di tutti i saggi di questa raccolta, Alan ENGLISH (Verlaine: Blurred Images and Ambiguous Text, pp. 123-136) evidenzia che la composizione delle opere di Verlaine coincide rudemente con la rivoluzione impressionista. Ne sono un esempio gli anni tra il 1860 ed il 1870 , che testimoniano una certa convergenza di finalità tra lo scritto e le arti visive; un risultato, forse, del comune spirito contestatario che ha caratterizzato diverse aree dell'attività umana in Francia, come la politica, la scienza e la società.

David DANBY (Gesture, Point of View and Proto-cinema in Victor Hugo's “Les Misérables", pp. 137-155) prende in esame altri elementi visivi: i gesti, la fisionomia e lo sguardo; tutti resi con successo nelle frasi hugoliane per mezzo di una tecnica linguistica spesso utilizzata per animare la scena. L'A. nota come la descrizione dei movimenti del corpo è usata da Hugo per diversi scopi: rappresentare il punto di vista, dipingere il significato affettivo ed esternare i pensieri interiori.

Secondo Nicolas fÈvE (Rhétorique de la photographie dans l'autobiographie contemporaine: "Des Histoires vraies" de Sophie Calle, pp. 157-170) tra il testo e la fotografia vi è un alto grado di complementarietà: l'immagine illustra il testo o può essere vista come una rivelazione del testo, così come, allo stesso modo, il testo può essere visto come una rivelazione, o possibilmente un'espressione, della fotografia. All'interno di questa complementarietà, ci sono, comunque, esempi in cui testo e immagine svolgono 
funzioni opposte: la fotografia è in grado di mostrare significati nascosti ed è vicina all'inconscio; il testo è comunque espressione conscia, la quale può anche descrivere visivamente elementi non rappresentabili come il contesto.

11 Sull'opera di Daniel Pennac si focalizza l'attenzione di Sarah cant (Image, Word, Absence and Silence in Pennac's "Monsieur Malaussène", pp. 171-179). Diverse rappresentazioni di immagine nella società e, in particolare, della relazione dell'immagine con il linguaggio, caratterizzano la dinamica centrale del testo di Pennac, il cui plot è costruito su un attento ed intricato intreccio di parola e immagine.

Il volume si chiude con il saggio di Nicolas WANLIN (Description as the Manifestation of Absence: Analysis of Pictoriality in Aloysius Bertrand's "Gaspard de la nuit", pp. 181-188) che ruota intorno all'idea della presenza e dell'assenza, in cui hypotyposis e ekphrasis vengono messe in discussione in un moderno contesto semiotico. Basandosi sul racconto Gaspard de la nuit, l'A. mostra come la presenza sensoriale dell'oggetto è "perduta", nella descrizione, attraverso la contaminazione culturale. 